TRANSACTIONS OF THE

AMERICAN MATHEMATICAL SOCIETY

Volume 358, Number 6, Pages 2651-2662

S 0002-9947(06)03913-4

Article electronically published on January 24, 2006

\title{
BOUNDED HOCHSCHILD COHOMOLOGY OF BANACH ALGEBRAS WITH A MATRIX-LIKE STRUCTURE
}

\author{
NIELS GRØNBÆK
}

\begin{abstract}
Let $\mathfrak{B}$ be a unital Banach algebra. A projection in $\mathfrak{B}$ which is equivalent to the identitity may give rise to a matrix-like structure on any two-sided ideal $\mathfrak{A}$ in $\mathfrak{B}$. In this set-up we prove a theorem to the effect that the bounded cohomology $\mathcal{H}^{n}\left(\mathfrak{A}, \mathfrak{A}^{*}\right)$ vanishes for all $n \geq 1$. The hypotheses of this theorem involve (i) strong H-unitality of $\mathfrak{A}$, (ii) a growth condition on diagonal matrices in $\mathfrak{A}$, and (iii) an extension of $\mathfrak{A}$ in $\mathfrak{B}$ by an amenable Banach algebra. As a corollary we show that if $X$ is an infinite dimensional Banach space with the bounded approximation property, $L_{1}(\mu, \Omega)$ is an infinite dimensional $L_{1}$-space, and $\mathfrak{A}$ is the Banach algebra of approximable operators on $L_{p}(X, \mu, \Omega)(1 \leq p<\infty)$, then $\mathcal{H}^{n}\left(\mathfrak{A}, \mathfrak{A}^{*}\right)=(0)$ for all $n \geq 0$.
\end{abstract}

\section{INTRODUCTION}

Recall that a Banach algebra $\mathfrak{A}$ is called weakly amenable if bounded derivations $D: \mathfrak{A} \rightarrow \mathfrak{A}^{*}$ are all inner. Weak amenability of Banach algebras has been the object of many studies. Recent studies have focused on algebras of compact, in particular approximable, operators on Banach spaces. In DGG it is shown that if $Y$ is a reflexive Banach space with the approximation property, then $\mathcal{A}\left(\ell_{p}(Y)\right), 1<p<$ $\infty$, is weakly amenable. In [B2, Theorem 4.1] it is shown that if $E$ is a Banach space such that the dual space $E^{*}$ has the bounded approximation property and $(\Omega, \Sigma, \mu)$ is a measure space such that $L_{p}(\mu)$ is infinite dimensional, then $\mathcal{A}\left(L_{p}(\mu, E)\right), 1 \leq$ $p<\infty$, is weakly amenable. In order to prove this (and many other results on weak amenability) Blanco introduced the so-called trace unbounded systems. The crucial technical step in these proofs consists of taking certain averages with respect to such trace-unbounded systems by exploiting a matrix-like structure. In G3] we have shown that $\mathcal{A}\left(L_{p}(\mu, E)\right), 1 \leq p<\infty$, is weakly amenable under the relaxed assumption (actually both a necessary and sufficient condition) that $\mathcal{A}\left(L_{p}(\mu, E)\right)$ ) is self-induced. Loosely speaking this means that multiplication is an isomorphism as a balanced bilinear map. In proving this we made use of the matrix-like structure provided by the right and left shifts on $\ell_{p}(E)$.

The importance of self-inducedness is that it allows for extending derivations to enveloping algebras. The definition of self-inducedness (see [G1]) is equivalent to the part of Wodzicki's definition of H-unitality (W1]) involving degree 1. Since weak amenability can be described as the property $\mathcal{H}^{1}\left(\mathfrak{A}, \mathfrak{A}^{*}\right)=\{0\}$, it is natural to investigate the relation between $\mathrm{H}$-unitality and the vanishing of higher cohomology

Received by the editors December 2, 2003 and, in revised form, August 3, 2004.

2000 Mathematics Subject Classification. Primary 46M20; Secondary 47B07, 16E40.

Key words and phrases. Bounded Hochschild cohomology, H-unital, simplicially trivial.

(C)2006 American Mathematical Society

Reverts to public domain 28 years from publication 
groups $\mathcal{H}^{n}\left(\mathfrak{A}, \mathfrak{A}^{*}\right)=\{0\}$. This is precisely the concern of the present paper. Specific instances of the question of vanishing of higher cohomology have been of great importance, notably the result that $\mathcal{H}^{n}\left(\mathfrak{A}, \mathfrak{A}^{*}\right)=\{0\}, n \geq 0$, for all $C^{*}$-algebras $\mathfrak{A}$ without bounded traces $\mathrm{CS}$, and the proof of the confirmative answer to the "additive" Karoubi conjecture which simultaneously gives that $\mathcal{H}^{n}\left(\mathfrak{A}, \mathfrak{A}^{*}\right)=\{0\}, n \geq 0$, for stable $C^{*}$-algebras $\mathfrak{A}$; see W2. For Banach algebras of operators on general Banach spaces not much is known. The proof in W2 that $\mathcal{B}(H)$, the bounded operators on a Hilbert space, has $\mathcal{H}^{n}\left(\mathcal{B}(H), \mathcal{B}(H)^{*}\right)=\{0\}, n \geq 0$, can easily be adapted to replace $H$ with $\ell_{p}(X)$, for arbitrary Banach spaces $X$. However, the method relies on the possibility to make infinite amplifications of operators, using $H \sim \bigoplus_{1}^{\infty} H$ and thus cannot be adapted to algebras of compact operators. Our investigations are partly aimed at circumventing this problem. We prove the following theorem:

Theorem. Let $X$ be a Banach space, let $1 \leq p<\infty$ and suppose that the algebra of approximable operators $\mathcal{A}\left(\ell_{p}(X)\right)$ is strongly $H$-unital as a Banach algebra. Then it is simplicially trivial, i.e., $\mathcal{H}^{n}\left(\mathcal{A}\left(\ell_{p}(X)\right), \mathcal{A}\left(\ell_{p}(X)^{*}\right)\right)=\{0\}$ for all $n \geq 0$.

As a corollary we show that all $\mathcal{A}\left(L_{p}(\mu, X)\right), 1 \leq p<\infty$, are simplicially trivial, when $L_{p}(\mu)$ is infinite dimensional and $X$ has the bounded approximation property.

The Banach algebra $\mathcal{A}\left(\ell_{p}(X)\right)$ may be viewed as a topological tensor product $\mathcal{A}\left(\ell_{p}\right) \bar{\otimes} \mathcal{A}(X)$, that is, $\mathcal{A}\left(\ell_{p}(X)\right)$ is reminiscent of a stabilization in $C^{*}$-algebra theory. It is therefore natural to expect that, in combination with $\mathrm{H}$-unitality, the (co)homology is trivial. As Wodzicki's result uses specific $C^{*}$-properties, we cannot appeal directly to his approach. Instead, we use that a dense subalgebra has trivial algebraic homology and extend this by continuity, using elementary chain homotopy techniques.

\section{THE SET-UP}

We start by describing the Hochschild homological setting in which we will be working. We appeal to preliminary knowledge and will be rather brief.

Let $A$ be an algebra (not necessarily unital) over $\mathbb{C}$. We define $n$-chains $C_{n}(A)$ and face maps $d_{i}: C_{n}(A) \rightarrow C_{n-1}(A), i=0, \ldots, n$, as

$$
C_{n}(A)=A^{\otimes(n+1)}
$$

and

$$
d_{i}\left(a_{0} \otimes \cdots \otimes a_{n}\right)= \begin{cases}a_{0} \otimes \cdots \otimes a_{i} a_{i+1} \otimes \cdots \otimes a_{n}, & i=0, \ldots, n-1, \\ a_{n} a_{0} \otimes \cdots \otimes a_{n-1}, & i=n .\end{cases}
$$

We consider the bar-complex

$$
B_{\bullet}(A): \quad 0 \leftarrow A \stackrel{\beta}{\longleftarrow} A \otimes A \stackrel{\beta}{\longleftarrow} \ldots \stackrel{\beta}{\longleftarrow} A^{\otimes n} \stackrel{\beta}{\longleftarrow} A^{\otimes(n+1)} \ldots,
$$

with the bar-differential on $n$-chains as

$$
\beta=\sum_{i=0}^{n-1}(-1)^{i} d_{i}
$$

Likewise, we have the Hochschild complex

$$
C_{\bullet}(A): \quad 0 \leftarrow A \stackrel{\delta}{\longleftarrow} A \otimes A \stackrel{\delta}{\longleftarrow} \ldots \stackrel{\delta}{\longleftarrow} A^{\otimes n} \stackrel{\delta}{\longleftarrow} A^{\otimes(n+1)} \ldots,
$$


with the Hochschild differential on $n$-chains as

$$
\delta=\sum_{i=0}^{n}(-1)^{i} d_{i}=\beta+(-1)^{n} d_{n} .
$$

For a Banach algebra we consider the corresponding bounded complexes based on the projective tensor product,

$$
\text { B. }(\mathfrak{A}): \quad 0 \leftarrow \mathfrak{A} \stackrel{\beta}{\longleftarrow} \mathfrak{A} \widehat{\otimes} \mathfrak{A} \stackrel{\beta}{\longleftarrow} \ldots \stackrel{\beta}{\longleftarrow} \mathfrak{A}^{\widehat{\otimes} n} \stackrel{\beta}{\longleftarrow} \mathfrak{A}^{\widehat{\otimes}(n+1)} \ldots,
$$

and

$$
\text { C. }(\mathfrak{A}): \quad 0 \leftarrow \mathfrak{A} \stackrel{\delta}{\longleftarrow} \mathfrak{A} \widehat{\otimes} \mathfrak{A} \stackrel{\delta}{\longleftarrow} \ldots \stackrel{\delta}{\longleftarrow} \mathfrak{A}^{\widehat{\otimes} n} \stackrel{\delta}{\longleftarrow} \mathfrak{A}^{\widehat{\otimes}(n+1)} \ldots,
$$

with $n$-chains $\mathbf{C}_{n}(\mathfrak{A})=\mathfrak{A}^{\widehat{\otimes}(n+1)}$ and the same defining formulas for face maps $d_{i}$ and differentials $\beta$ and $\delta$. Upon taking homology of the complexes $C_{\bullet}(A)$ and $\mathbf{C}_{\bullet}(\mathfrak{A})$ we arrive at the Hochschild homology groups $H_{n}(A, A), n=0,1, \ldots$, respectively, the bounded Hochschild homology groups $\mathcal{H}_{n}(\mathfrak{A}, \mathfrak{A}), n=0,1, \ldots$

The duals of the complexes give co-complexes and corresponding cohomology. Specifically, we have

$$
C^{\bullet}(A): \quad 0 \rightarrow A^{\prime} \stackrel{\partial}{\longrightarrow}(A \otimes A)^{\prime} \stackrel{\partial}{\longrightarrow} \ldots \stackrel{\partial}{\longrightarrow}\left(A^{\otimes n}\right)^{\prime} \stackrel{\partial}{\longrightarrow}\left(A^{\otimes(n+1)}\right)^{\prime} \ldots,
$$

and

$$
\mathbf{C}^{\bullet}(\mathfrak{A}): \quad 0 \rightarrow \mathfrak{A}^{*} \stackrel{\partial}{\longrightarrow}(\mathfrak{A} \widehat{\otimes} \mathfrak{A})^{*} \stackrel{\partial}{\longrightarrow} \ldots \stackrel{\partial}{\longrightarrow}\left(\mathfrak{A}^{\widehat{\otimes} n}\right)^{*} \stackrel{\partial}{\longrightarrow}\left(\mathfrak{A}^{\hat{\otimes}(n+1)}\right)^{*} \ldots,
$$

where $(\cdot)^{\prime}$ denotes algebraic dual and $(\cdot)^{*}$ denotes topological dual. We have used $\partial$ as the common symbol for algebraic and topological dual maps of the likewise commonly denoted Hochschild differentials. Correspondingly, we have the Hochschild cohomology groups $H^{n}\left(A, A^{\prime}\right)$ and the bounded Hochschild cohomology groups $\mathcal{H}^{n}\left(\mathfrak{A}, \mathfrak{A}^{*}\right)$.

We now define the central notion of H-unitality due to M. Wodzicki W1].

1.1 Definitions. A complex algebra $A$ is $H$-unital if the bar-complex $B_{\bullet}(A)$ is acyclic. A Banach algebra $\mathfrak{A}$ is strongly $H$-unital if $\mathbf{B} \cdot(\mathfrak{A})$ is pure exact, i.e., $X \widehat{\otimes} \mathbf{B} \cdot(\mathfrak{A})$ is acyclic for all Banach spaces $X$. The Banach algebra $\mathfrak{A}$ is simplicially trivial if $\mathcal{H}^{n}\left(\mathfrak{A}, \mathfrak{A}^{*}\right)=\{0\}$ for all $n \geq 0$.

The importance of H-unitality is that it is the condition for excision in Hochschild (co)homology. Our strategy shall be to use excision according to a scheme

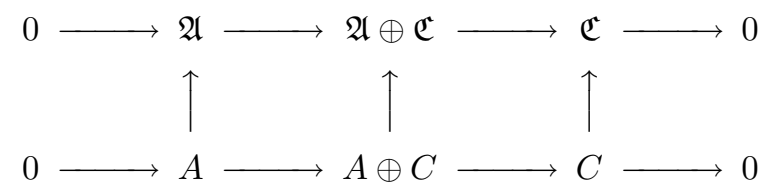

where we have control of the algebraic cohomology in the bottom row and use continuity to extend it to the top row. This is done by exploiting matrix structures (tailored to deal with Banach algebras of operators on Banach spaces of vectorvalued sequences) which we now discuss.

Assume that the Banach algebra $\mathfrak{A}$ has bounded multipliers $S$ and $R$ such that $R S=\mathbf{1}_{\mathfrak{A}}$ and $S R \neq \mathbf{1}_{\mathfrak{A}}$. Let $\mathfrak{A}_{k}=\left\{a \in \mathfrak{A} \mid R^{k} a=a S^{k}=0\right\}$. Then $\mathfrak{A}_{1} \subseteq \mathfrak{A}_{2} \subseteq \ldots$. 
Let $P_{i}=S^{i-1} R^{i-1}-S^{i} R^{i}, i=1,2, \ldots$ Then $P_{i} \neq 0, i=1,2, \ldots$, and each element in $\mathfrak{A}_{k}$ may be represented as an infinite upper left corner matrix

$$
\left(a_{i j}\right)=\left(\begin{array}{ccccc}
a_{11} & \ldots & a_{1 k} & 0 & \ldots \\
\vdots & \ddots & \vdots & \vdots & \ldots \\
a_{k 1} & \ldots & a_{k k} & 0 & \ldots \\
0 & \ldots & 0 & 0 & \ldots \\
\vdots & & \vdots & &
\end{array}\right)
$$

with $a_{i j}=P_{i} a P_{j}$ for some $a \in \mathfrak{A}$. In this picture multiplication in $\mathfrak{A}_{k}$ is just matrix multiplication.

Condition M1. If the algebra of "upper left-hand corners" $\mathfrak{A}_{\infty}$, given by

$$
\mathfrak{A}_{\infty}=\bigcup_{1}^{\infty} \mathfrak{A}_{k}
$$

is dense in $\mathfrak{A}$, and if "block diagonal" matrices are bounded, that is, for each $a \in \mathfrak{A}_{\infty}$,

$$
\nu(a):=\sup \left\{\left\|\sum_{l=0}^{N} S^{l k} a R^{l k}\right\|\right\}<\infty,
$$

where the supremum is taken over all $N \in \mathbb{N}$ and all $k \in \mathbb{N}$ such that $a \in \mathfrak{A}_{k}$, then $\mathfrak{A}$ satisfies Condition M1 w.r.t. $S$ and $R$.

In order to lift cohomology from a dense subalgebra we shall further be interested in

Condition M2. If there is a dense subalgebra $A \subseteq \mathfrak{A}$ such that with $A_{k}=\{a \in$ $\left.A \mid R^{k} a=a S^{k}=0\right\}=A \cap \mathfrak{A}_{k}$, the subalgebra

$$
A_{\infty}=\bigcup_{1}^{\infty} A_{k}
$$

is H-unital and $H_{n}\left(A_{\infty}, A_{\infty}^{\prime}\right)=\{0\}, n \geq 1$, then $\mathfrak{A}$ satisfies Condition M2 w.r.t. $S$ and $R$.

From the matrix picture of $\mathfrak{A}_{\infty}$ it follows that scalar matrices may operate on $\mathfrak{A}_{\infty}$ by means of matrix multiplication. The purpose in our setting of requiring H-unitality is that excision enables us to extend cocycles to certain operations of permutations. This leads to the requirement

Condition M3. Let $\mathfrak{S}(\mathbb{N})$ be the full permutation group of $\mathbb{N}$ and consider the subgroup

$$
\mathfrak{G}=\{\mathfrak{p} \in \mathfrak{S}(\mathbb{N}) \mid \mathfrak{p}(i) \neq i \text { for at most finitely many } i \in \mathbb{N}\}
$$

of finite permutations of $\mathbb{N}$. Let $\boldsymbol{D}$ be the group of infinite diagonal scalar matrices with -1 in at most finitely many diagonal entries and +1 in the remaining diagonal entries and let $\mathcal{U}$ be the group of infinite matrices of the form $U=\mathrm{D} \Pi_{\mathfrak{p}}$, where $\mathrm{D} \in \boldsymbol{D}$ and $\Pi_{\mathfrak{p}}$ is the permutation matrix corresponding to some $\mathfrak{p} \in \mathfrak{G}$. We define the multiplier norm of $U \in \mathcal{U}$ as

$$
\|U\|=\sup \left\{\left\|\left(a_{i j}\right) U\right\|,\left\|U\left(a_{i j}\right)\right\| \mid\left(a_{i j}\right) \in \mathfrak{A}_{\infty},\left\|\left(a_{i j}\right)\right\| \leq 1\right\} .
$$


If $\kappa(\mathcal{U}):=\sup \left\{\|U\|\left\|U^{-1}\right\| \mid U \in \mathcal{U}\right\}<\infty$, then $\mathfrak{A}$ satisfies Condition M3 w.r.t. $S$ and $R$.

Note that for each $\mathfrak{p} \in \mathfrak{G}$ and each diagonal matrix $\mathrm{D} \in \boldsymbol{D}$ there is $\mathrm{D}^{\prime} \in \boldsymbol{D}$ such that $\mathrm{D} \Pi_{\mathfrak{p}}=\Pi_{\mathfrak{p}} \mathrm{D}^{\prime}$.

We finish this section by observing some consequences of parts of Conditions M1, M2 and M3.

1.2 Proposition. Suppose that $S$ and $R$ are bounded multipliers of a Banach algebra $\mathfrak{A}$ with $R S=\mathbf{1}_{\mathfrak{A}}$ and $S R \neq \mathbf{1}_{\mathfrak{A}}$. With the notation as above, if $\mathfrak{A}_{\infty}$ is dense in $\mathfrak{A}$ and $\kappa(\mathcal{U})<\infty$, then:

(i) Each $U \in \mathcal{U}$ can be extended by continuity to a bounded multiplier on $\mathfrak{A}$.

(ii) The idempotents $S^{n} R^{n}, n \in \mathbb{N}$, are uniformly bounded in multiplier norm. It further follows that

(iii) $\left\|S^{n} R^{n} a\right\|+\left\|a S^{n} R^{n}\right\| \rightarrow 0$ as $n \rightarrow \infty$ for all $a \in \mathfrak{A}$.

(iv) $A_{\infty}$ is dense in $\mathfrak{A}$.

Conversely, if $\mathfrak{A}$ satisfies (iii), then (ii) and (iv) hold. In particular, $\mathfrak{A}_{\infty}$ is dense in $\mathfrak{A}$.

Proof. Since $\mathfrak{A}_{\infty}$ is dense, (i) is obvious. The group $\boldsymbol{D}$ of matrices is bounded by $\sqrt{\kappa(\mathcal{U})}$, since each of its elements has order 2. Each of the idempotents $S^{n} R^{n}$ has a decomposition $S^{n} R^{n}=\frac{1}{2}(\mathbf{1}-\mathrm{D})$ where $\mathrm{D} \in \boldsymbol{D}$ is the diagonal matrix with -1 in the first $n$ entries and 1 in the remaining, so $S^{n} R^{n}, n \in \mathbb{N}$, is uniformly bounded in the multiplier norm. For each $a \in \mathfrak{A}_{\infty}$ the sequences $\left(S^{n} R^{n} a\right)$ and $\left(a S^{n} R^{n}\right)$ are eventually equal to 0 . By uniform boundedness and density of $\mathfrak{A}_{\infty}$ (iii) follows. Similarly, $P_{n} a P_{n} \rightarrow a$ uniformly on the unit ball of $\mathfrak{A}$. For $k \in \mathbb{N}$ we have $\mathfrak{A}_{k}=P_{k} \mathfrak{A} P_{k}$ and $A_{k}=P_{k} A P_{k}$, so density of $\mathfrak{A}_{\infty}$ and of $A$ imply density of $A_{\infty}$

If (iii) holds, then $\left\{S^{n} R^{n} \mid n \in \mathbb{N}\right\}$ is uniformly bounded in the multiplier norm by the uniform boundedness principle. We see as above that if $A$ is dense in $\mathfrak{A}$, then $A_{\infty}$ is dense in $\mathfrak{A}$.

\section{THE THEOREM}

2.1 Theorem. Let $\mathfrak{A}$ be a strongly H-unital Banach algebra. If there are multipliers $S$ and $R$ such that $\mathfrak{A}$ satisfies Conditions $\mathrm{M} 1, \mathrm{M} 2$, and $\mathrm{M} 3$ w.r.t. $S$ and $R$, then

$$
\mathcal{H}^{n}\left(\mathfrak{A}, \mathfrak{A}^{*}\right)=\{0\}, \quad n \geq 1 .
$$

The proof of this will occupy the next two sections. Thus we shall assume throughout that $\mathfrak{A}$ satisfies the hypotheses. First we make some reductions of the problem.

Let $D \in \mathbf{C}^{n}(\mathfrak{A})$ be an $n$-cocycle, i.e., $D$ is a bounded linear functional on $\mathfrak{A} \widehat{\otimes} \cdots \widehat{\otimes} \mathfrak{A}$ (equivalently, a bounded $(n+1)$-linear functional on $\mathfrak{A})$ such that $\partial D=0$. By the first part of Condition M2 there is a (not necessarily bounded) $n$-linear functional $\phi$ such that

$$
D\left(a_{0} \otimes \cdots \otimes a_{n}\right)=\phi\left(\delta\left(a_{0} \otimes \cdots \otimes a_{n}\right)\right), \quad a_{i} \in A_{\infty}, i=0, \ldots, n .
$$

Since $A_{\infty}$ is dense in $\mathfrak{A}$, we may prove that $D$ is a (bounded) coboundary by proving that $\phi$ can be chosen to be bounded on $\delta\left(A_{\infty} \otimes \cdots \otimes A_{\infty}\right)$. First we exploit $\mathrm{H}$-unitality. By Proposition 1.2 we may regard $\mathcal{U}$ as a group of invertible operators 
in $\mathcal{B}(\mathfrak{A})$. As in the proof of [J] Proposition 6.1] we see that the group $\mathcal{U}$ is amenable. With $\omega(U)=\|U\|$ the weighted convolution algebra $\ell_{1}(\mathcal{U}, \omega)$ is amenable by the main result of [G4], since $\kappa(\mathcal{U})<\infty$. Let $\mathfrak{C}$ be the Banach subalgebra of $\mathcal{B}(\mathfrak{A})$ generated by $\mathcal{U}$. Containing a dense continuous image of an amenable Banach algebra $\mathfrak{C}$ is itself amenable.

Consider the extension

$$
0 \rightarrow \mathfrak{A} \stackrel{\iota}{\longrightarrow} \mathfrak{A} \oplus \mathfrak{C} \stackrel{q}{\longrightarrow} \mathfrak{C} \rightarrow 0,
$$

where $\mathfrak{A} \oplus \mathfrak{C}$ is the semi-direct product with multiplication given by the multiplier action of $\mathfrak{C}$ on $\mathfrak{A}$.

2.2 Proposition. Assume that $\mathfrak{A}$ is strongly $H$-unital. Then every bounded $n$ cocycle may be extended to a bounded n-cocycle on the semi-direct product $\mathfrak{A} \oplus \mathfrak{C}$.

Proof. Set $\mathfrak{B}=\mathfrak{A} \oplus \mathfrak{C}$. By W1] we get a long exact sequence from (2)

$$
\ldots \rightarrow \mathcal{H}_{n}(\mathfrak{A}, \mathfrak{A}) \stackrel{\iota_{*}}{\longrightarrow} \mathcal{H}_{n}(\mathfrak{B}, \mathfrak{B}) \rightarrow \mathcal{H}_{n}(\mathfrak{C}, \mathfrak{C}) \rightarrow \mathcal{H}_{n-1}(\mathfrak{A}, \mathfrak{A}) \stackrel{\iota_{*}}{\longrightarrow} \cdots
$$

By amenability $\mathcal{H}_{n}(\mathfrak{C}, \mathfrak{C})=\{0\}, n \geq 1$ so that the inclusion $\mathfrak{A} \stackrel{\iota}{\longrightarrow} \mathfrak{B}$ induces an isomorphism in bounded homology. Hence the restriction map induces an isomorphism in bounded cohomology, i.e., for $n \geq 1$ every bounded $n$-cocycle on $\mathfrak{A}$ is the restriction of a bounded $n$-cocycle on $\mathfrak{B}$.

We shall also need (a consequence of) the discrete version of this theorem. We let $M_{\infty}$ denote the algebra of infinite matrices over $\mathbb{C}$ consisting of "upper left-hand corners", $M_{\infty}=\left\{\left(\lambda_{i j}\right) \mid \exists k \in \mathbb{N}:(i \geq k\right.$ or $\left.j \geq k) \Longrightarrow \lambda_{i j}=0\right\}$. We further define $\widetilde{M}_{\infty}=M_{\infty} \oplus \mathfrak{D}$, where $\mathfrak{D}$ is the algebra of infinite diagonal matrices with constant diagonal, so that we have an augmentation

$$
0 \rightarrow M_{\infty} \rightarrow \widetilde{M}_{\infty} \rightarrow \mathbb{C} \rightarrow 0
$$

As above we may regard $\widetilde{M}_{\infty}$ as multipliers on $\mathfrak{A}$. In fact, $\widetilde{M}_{\infty}$ is a subalgebra of $\mathfrak{C}$. We set $B=A_{\infty} \oplus \widetilde{M}_{\infty}$. Then

2.3 Proposition. $H_{n}(B, B)=H^{n}\left(B, B^{\prime}\right)=\{0\}$ for all $n \geq 1$.

Proof. Consider the extension

$$
0 \rightarrow A_{\infty} \rightarrow B \rightarrow \widetilde{M}_{\infty} \rightarrow 0 .
$$

By M2 $A_{\infty}$ is H-unital so that we have excision in discrete homology, that is, we have a long exact sequence

(6) $\ldots \rightarrow H_{n}\left(A_{\infty}, A_{\infty}\right) \rightarrow H_{n}(B, B) \rightarrow H_{n}\left(\widetilde{M}_{\infty}, \widetilde{M}_{\infty}\right) \rightarrow H_{n-1}\left(A_{\infty}, A_{\infty}\right) \rightarrow \ldots$

The tensor trace is a quasi-isomorphism [L, Theorem 1.2.4], so we get $H_{n}\left(M_{\infty}, M_{\infty}\right)$ $=H_{n}(\mathbb{C}, \mathbb{C})=\{0\}$. Having local units $M_{\infty}$ is H-unital (see Proposition 4.1 (ii)), yielding $H_{n}\left(\widetilde{M}_{\infty}, \widetilde{M}_{\infty}\right)=\{0\}, n \geq 0$. By the assumption $\mathrm{M} 2, H_{n}\left(A_{\infty}, A_{\infty}\right)=$ $\{0\}, n \geq 1$, so excision gives the desired result for homology. Observing that in general a sequence of vector spaces and linear maps $X \stackrel{s}{\longrightarrow} Y \stackrel{t}{\longrightarrow} Z$ is exact at $Y$ if and only if the algebraic dual sequence $X^{\prime} \stackrel{s^{\prime}}{\longleftarrow} Y^{\prime} \stackrel{t^{\prime}}{\longleftarrow} Z^{\prime}$ is exact at $Y^{\prime}$, also $H^{n}\left(B, B^{\prime}\right)=\{0\}$.

We return to the bounded $n$-cocycle $D$. We collect the above findings in the following proposition. 
2.4 Proposition. Assume that $\mathfrak{A}$ is strongly $H$-unital, and let $D$ be a bounded $n$-cocycle on $\mathfrak{A}$. Then there is a bounded $n$-cocycle $\widetilde{D}$ on $\mathfrak{B}=\mathfrak{A} \oplus \mathfrak{C}$ extending $D$ and there is a (not necessarily bounded) n-linear functional $\widetilde{\phi}$ on $B=A_{\infty} \oplus \widetilde{M}_{\infty}$, such that

$$
\widetilde{D}\left(b_{0} \otimes \cdots \otimes b_{n}\right)=\widetilde{\phi}\left(\delta\left(b_{0} \otimes \cdots \otimes b_{n}\right)\right), b_{0}, \ldots, b_{n} \in B
$$

\section{THE PROOF}

In the following we drop the ${ }^{\sim}$ s, and hence consider $D$ to be a bounded $n$-cocycle on $\mathfrak{B}$ with corresponding $n$-linear functional $\phi$ on $B$. By [JKR, Theorem 4.1] we may further assume that $D$ is normalized with respect to the amenable subalgebra $\mathfrak{C}$ of $\mathfrak{B}$, that is,

$$
D\left(b_{0} \otimes \cdots \otimes b_{n}\right)=0 \text { if any of } b_{0}, \ldots, b_{n} \text { lies in } \mathfrak{C} .
$$

For each $k \in \mathbb{N}$ we define a bounded grade zero chain map $\sigma_{k}: \mathbf{C} .(\mathfrak{A}) \rightarrow \mathbf{C} \bullet(\mathfrak{A})$ by

$$
\sigma_{k}\left(a_{0} \otimes \cdots \otimes a_{n}\right)=S^{k} a_{0} R^{k} \otimes \cdots \otimes S^{k} a_{n} R^{k} .
$$

The first step in proving that $D$ is a bounded coboundary on $\mathfrak{A}$ is

3.1 Proposition. Let $\tau$ be an $(n-1)$-cycle in $C_{n-1}\left(A_{\infty}\right)$. For $k \in \mathbb{N}$ such that $\tau \in A_{k}^{\otimes n}$

$$
\phi\left(\sigma_{k}(\tau)\right)=\phi(\tau)
$$

Proof. Let $U \in \mathfrak{C}$ be the unitary order 2 operator given by the permutation $(1 k+1) \circ(2 k+2) \circ \cdots \circ(k 2 k)$ of coordinates. Then

$$
S^{k} a R^{k}=U a U^{-1}, a \in A_{k} .
$$

Since $U$ is of order 2 , we may write $U=e^{h}$ with $h=\frac{i \pi}{2}(U-\mathbf{1})$. We shall use a typical homotopy argument to finish. Define a bounded chain map $\alpha_{t}: \mathbf{C} \bullet(\mathfrak{A}) \rightarrow$ C.(A) by

$$
\alpha_{t}\left(a_{0} \otimes \cdots \otimes a_{n}\right)=e^{t h} a_{0} e^{-t h} \otimes \cdots \otimes e^{t h} a_{n} e^{-t h} .
$$

Then $\alpha_{0}(\tau)=\tau$ and $\alpha_{1}(\tau)=\sigma_{k}(\tau)$. We further have

$$
\frac{d}{d t} \alpha_{t}(\tau)=\alpha_{t}\left(\Gamma_{h}(\tau)\right)
$$

where

$$
\Gamma_{h}\left(a_{0} \otimes \cdots \otimes a_{n}\right)=\sum_{i=0}^{n} \ldots \otimes\left[h a_{i}-a_{i} h\right] \otimes \ldots
$$

It is well known $\left[\mathrm{L}\right.$, Proposition 1.3.3] that $\Gamma_{h}$ is 0-homotopic. A bounded contracting chain homotopy $s: \mathbf{C} \bullet(\mathfrak{B}) \rightarrow \mathbf{C}_{\bullet+1}(\mathfrak{B})$ is given by

$$
s\left(a_{0} \otimes \cdots \otimes a_{n}\right)=\sum_{k=0}^{n}(-1)^{k+1} a_{0} \otimes \cdots \otimes a_{k} \otimes h \otimes a_{k+1} \otimes \cdots \otimes a_{n} .
$$


Hence

$$
\begin{aligned}
\sigma_{k}(\tau)-\tau & =\int_{0}^{1} \frac{d}{d t} \alpha_{t}(\tau) d t \\
& =\int_{0}^{1} \alpha_{t}\left(\Gamma_{h}(\tau)\right) d t \\
& =\int_{0}^{1} \alpha_{t}((s \delta+\delta s) \tau) d t \\
& =\delta \int_{0}^{1} \alpha_{t}(s(\tau)) d t
\end{aligned}
$$

where the last step follows from $\delta \tau=0$ and elementary properties of the Bochner integral. Note that $\int_{0}^{1} \alpha_{t}(\tau) d t \in C_{n-1}\left(A_{\infty}\right)$ and that $\alpha_{t}(s(\tau)) \in C_{n}(B), t \in[0,1]$, is composed of elementary tensors with at least one tensor factor in $\mathfrak{C}$. On applying $\phi$ we get

$$
\begin{aligned}
\phi\left(\sigma_{k}(\tau)\right)-\phi(\tau) & =\phi\left(\delta \int_{0}^{1} \alpha_{t}(s(\tau)) d t\right) \\
& =D\left(\int_{0}^{1} \alpha_{t}(s(\tau)) d t\right) \\
& =\int_{0}^{1} D\left(\alpha_{t}(s(\tau))\right) d t \\
& =0
\end{aligned}
$$

where the last equality follows from $D$ being normalized w.r.t. $\mathfrak{C}$.

We are now ready for

Proof of Theorem 2.1. We shall prove that $D$, being normalized w.r.t. $\mathfrak{C}$, is actually identically zero. In doing this we shall make use of certain chain homotopies. For the rest of the proof we fix $k \in \mathbb{N}$. We first define certain graded maps. For each $N \in \mathbb{N}$ we define a grade 0 map $\Delta_{N}: \mathbf{C} .(\mathfrak{B}) \rightarrow$ C. $(\mathfrak{B})$ by

$$
\Delta_{N}\left(a_{0} \otimes \cdots \otimes a_{n}\right)=\sum_{l=0}^{N-1} S^{l k} a_{0} R^{l k} \otimes \cdots \otimes \sum_{l=0}^{N-1} S^{l k} a_{n} R^{l k},
$$

and for each $l=0, \ldots, N-1$ the grade 1 maps $h_{l, i}^{N}: \mathbf{C}_{\bullet}(\mathfrak{B}) \rightarrow \mathbf{C}_{\bullet+1}(\mathfrak{B})$ by

$$
h_{l, i}^{N}\left(a_{0} \otimes \cdots \otimes a_{n}\right)=\sigma_{l k}\left(a_{0} \otimes \cdots \otimes a_{i}\right) \otimes P_{l} \otimes \Delta_{N}\left(a_{i+1} \otimes \cdots \otimes a_{n}\right), i=0, \ldots, n,
$$

where $P_{l}=S^{l k} R^{l k}-S^{(l+1) k} R^{(l+1) k}$ is the projection onto the $(l+1)$-th coordinate block of size $k$.

Remarks. Note that $P_{l} \in \mathfrak{C}$, since it has the form $\frac{1}{2}\left(\mathrm{D}_{1}-\mathrm{D}_{2}\right)$ for appropriate $\mathrm{D}_{1}, \mathrm{D}_{2} \in \boldsymbol{D}$ and that the grade zero maps $\Delta_{N}$ can be described by means of the previously defined chain maps $\sigma_{l}$ :

$$
\Delta_{N}=\sum_{l=0}^{N-1} \sigma_{l k} \otimes \cdots \otimes \sum_{l=0}^{N-1} \sigma_{l k}
$$


The diagonal matrix picture below will be convenient. For $a_{0}, \ldots, a_{n} \in A_{k}$ we have

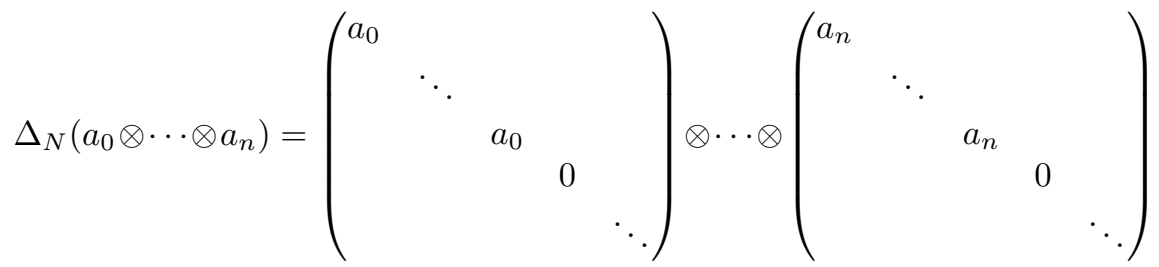

as upper left-hand $N \times N$ corners of $k \times k$ blocks. We observe that $\Delta_{N}$ is a chain map when restricted to $A_{k} \otimes \cdots \otimes A_{k}$ :

$$
\Delta_{N}\left(\delta\left(a_{0} \otimes \cdots \otimes a_{n}\right)\right)=\delta\left(\Delta_{N}\left(a_{0} \otimes \cdots \otimes a_{n}\right)\right), a_{0}, \ldots, a_{n} \in A_{k} .
$$

Furthermore,

$$
\begin{aligned}
h_{l, i}^{N}\left(a_{0} \otimes \cdots \otimes a_{n}\right) & \\
= & \left(\begin{array}{lllll}
0 & & & & \\
& \ddots & & & \\
& a_{0} & & \\
& & 0 & \\
& & & \ddots
\end{array}\right) \otimes \cdots \otimes\left(\begin{array}{ccccc}
0 & & & & \\
& \ddots & & & \\
& & \mathbf{1} & & \\
& & 0 & \\
& & & \ddots
\end{array}\right) \\
& \left(\begin{array}{cccccc}
a_{i+1} & & & & \\
& \ddots & & & \\
& & a_{i+1} & & \\
& & & 0 & \\
& & & & \ddots
\end{array}\right) \otimes\left(\begin{array}{lllll}
a_{n} & & & \\
& \ddots & & \\
& & a_{n} & & \\
& & & 0 & \\
& & & & \ddots
\end{array}\right),
\end{aligned}
$$

as upper left-hand $N \times N$ corners with $a_{0}, \ldots, a_{i}$ and $\mathbf{1}$ placed in the $(l+1)$-th diagonal $k \times k$ block entries.

For $a_{0}, \ldots, a_{n} \in A_{k}$ we have the relations

$$
d_{i} h_{l, j}^{N}\left(a_{0} \otimes \cdots \otimes a_{n}\right)= \begin{cases}h_{l, j-1}^{N} d_{i}\left(a_{0} \otimes \cdots \otimes a_{n}\right), & i<j, \\ d_{i} h_{l, i-1}^{N}\left(a_{0} \otimes \cdots \otimes a_{n}\right), & i=j, j+1, \\ h_{l, j}^{N} d_{i-1}\left(a_{0} \otimes \cdots \otimes a_{n}\right), & i>j+1 .\end{cases}
$$

For the 0 -th and $(n+1)$-th face maps we further have $d_{0} h_{l, 0}^{N}\left(a_{0} \otimes \cdots \otimes a_{n}\right)=$ $\sigma_{l k}\left(a_{0}\right) \otimes \Delta_{N}\left(a_{1} \otimes \cdots \otimes a_{n}\right)$ and $d_{n+1} h_{l, n}^{N}\left(a_{0} \otimes \cdots \otimes a_{n}\right)=\sigma_{l k}\left(a_{0} \otimes \cdots \otimes a_{n}\right)$. Setting

$$
h_{l}^{N}=\sum_{i=0}^{n}(-1)^{i} h_{l, i}^{N}
$$

gives the homotopy relations

(24) $\sigma_{l k}\left(a_{0} \otimes \cdots \otimes a_{n}\right)=\sigma_{l k}\left(a_{0}\right) \otimes \Delta_{N}\left(a_{1} \otimes \cdots \otimes a_{n}\right)+\left(\delta h_{l}^{N}+h_{l}^{N} \delta\right)\left(a_{0} \otimes \cdots \otimes a_{n}\right)$;

see Lemma 1.0.9 of $[\mathrm{L}$. Adding over $l$ finally gives the homotopy relation

$$
\sum_{l=0}^{N-1} \sigma_{l k}=\Delta_{N}+\delta\left(\sum_{l=0}^{N-1} h_{l}^{N}\right)+\left(\sum_{l=0}^{N-1} h_{l}^{N}\right) \delta,
$$


where we used $\sum_{l=0}^{N-1} \sigma_{l k}\left(a_{0}\right) \otimes \Delta_{N}\left(a_{1} \otimes \cdots \otimes a_{n}\right)=\Delta_{N}\left(a_{0} \otimes \cdots \otimes a_{n}\right)$. Applying this to $\delta \tau$ we get

$$
\frac{1}{N} \sum_{l=0}^{N-1}\left(\sigma_{l k}-\delta h_{l}^{N}\right)(\delta \tau)=\frac{1}{N} \Delta_{N}(\delta \tau)=\frac{1}{N} \delta\left(\Delta_{N}(\tau)\right),
$$

where the last equality follows from (20). In order to finish we use Condition M1 to establish the bound

$$
\nu(\tau):=\sup \left\{\left\|\Delta_{N}(\tau)\right\| \mid N \in \mathbb{N}\right\}<\infty .
$$

Since $\tau$ is a finite sum of elementary tensors from $A_{k}^{\otimes(n+1)}$, it suffices to show (27) for elementary tensors. For $a_{0}, \ldots, a_{n} \in A_{k}$,

$$
\left\|\Delta_{N}\left(a_{0} \otimes \cdots \otimes a_{n}\right)\right\|=\left\|\Delta_{N}\left(a_{0}\right)\right\| \cdots\left\|\Delta_{N}\left(a_{n}\right)\right\|
$$

so that, by Condition M1,

$$
\nu\left(a_{0} \otimes \cdots \otimes a_{n}\right) \leq \nu\left(a_{0}\right) \cdots \nu\left(a_{n}\right)<\infty .
$$

By the remarks after (17) the term $\sum_{l=0}^{N-1} h_{l}^{N}(\delta \tau)$ from (26) is a sum of elementary tensors with at least one tensor factor in $\mathfrak{C}$, so by normalization

$$
\phi\left(\sum_{l=0}^{N-1} \delta h_{l}^{N}(\delta \tau)\right)=D\left(\sum_{l=0}^{N-1} h_{l}^{N}(\delta \tau)\right)=0 .
$$

By Proposition 3.1, (26), and (30) we get

$$
\begin{aligned}
|\phi(\delta \tau)|=\mid \phi\left(\frac{1}{N} \sum_{l=0}^{N-1} \sigma_{l k}(\delta \tau) \mid\right. & =\left|\phi\left(\frac{1}{N} \delta\left(\Delta_{N}(\tau)\right)\right)\right| \\
& =\left|\frac{1}{N} D\left(\Delta_{N}(\tau)\right)\right| \\
& \leq \frac{1}{N}\|D\| \nu(\tau) .
\end{aligned}
$$

Letting $N \rightarrow \infty$ we get $\phi(\delta \tau)=D(\tau)=0$.

3.2 Remark. The use of $\mathrm{H}$-unitality has in essence only involved excision concerning extensions

$$
0 \rightarrow \mathfrak{A} \rightarrow \mathfrak{A} \oplus\langle U\rangle \rightarrow\langle U\rangle \rightarrow 0
$$

where $\langle U\rangle$ is a 2-dimensional Banach algebra with $U^{2}=1$.

\section{Some APplications}

First we note some well-known conditions to ensure partial fulfillment of Condition M2.

4.1 Proposition. For an algebra A:

(i) If $A$ has a splitting of the multiplication, that is, the multiplication map $\mu: A \otimes A \rightarrow A$ has an $A$-bimodule left inverse, then $A$ is $H$-unital and $H_{n}(A, A)=$ $H^{n}\left(A, A^{\prime}\right)=\{0\}$ for all $n \geq 1$.

(ii) If $A$ has local left or right units (i.e., for each finite set $\mathrm{M} \subseteq A$ there is $e_{\mathrm{M}} \in A$ so that $e_{\mathrm{M}} a=a, a \in \mathrm{M}$ or $\left.a e_{\mathrm{M}}=a, a \in \mathrm{M}\right)$, then $A$ is H-unital.

For a Banach algebra $\mathfrak{A}$ :

(iii) If $\mathfrak{A}$ has a bounded one-sided approximate identity, then $\mathfrak{A}$ is strongly $H$ unital. 
Proof. (i) If $\mathfrak{s}: A \rightarrow A \otimes A$ is a splitting of the multiplication, then $\mathfrak{s} \otimes \mathbf{1}: C \bullet(A) \rightarrow$ $C_{\bullet+1}(A)$ is a contracting homotopy of the Hochschild complex. The rest is contained in Proposition 2 and Proposition 5 of [W1].

Our first application is Banach algebras of compact operators on vector-valued sequence spaces. Let $X$ be a Banach space and let $\mathcal{A}(X)$ denote the Banach algebra of approximable operators on $X$. Since $\mathcal{A}(X)$ has no bounded traces, $\mathcal{H}^{0}\left(\mathcal{A}(X), \mathcal{A}(X)^{*}\right)=\{0\}$. For the Banach space of $p$-summable sequences, $\ell_{p}(X)$, $1 \leq p<\infty$, we have.

4.2 Theorem. If $\mathcal{A}\left(\ell_{p}(X)\right), 1 \leq p<\infty$, is strongly $H$-unital, then it is simplicially trivial.

Proof. We show that $\mathfrak{A}=\mathcal{A}\left(\ell_{p}(X)\right)$ satisfies Conditions M1, M2, and M3 with respect to the norm 1 shifts

$$
\begin{aligned}
& S\left(x_{1}, x_{2}, \ldots\right)=\left(0, x_{1}, x_{2}, \ldots\right), \\
& R\left(x_{1}, x_{2}, \ldots\right)=\left(x_{2}, x_{3}, \ldots\right), \quad\left(x_{1}, x_{2}, \ldots\right) \in \ell_{p}(X) .
\end{aligned}
$$

Let $A=\mathcal{F}\left(\ell_{p}(X)\right)$ be the algebra of finite rank operators on $\ell_{p}(X)$. Then, by a well-known construction, $A_{\infty}$ as defined in Condition M2, has a splitting of the multiplication: For $x \in X, x^{*} \in X^{*}$ and $i, j \in \mathbb{N}$ consider the rank 1 operator given by the elementary matrix $E_{i j}\left(x \otimes x^{*}\right)$. Then $A_{\infty}=\operatorname{span}\left\{E_{i j}\left(x \otimes x^{*}\right) \mid x \in\right.$ $\left.X, x^{*} \in X^{*}, i, j \in \mathbb{N}\right\}$. Choose $e \in X, e^{*} \in X^{*}$ with $e^{*}(e)=1$. A splitting of the multiplication is given by $E_{i j}\left(x \otimes x^{*}\right) \mapsto E_{i 1}\left(x \otimes e^{*}\right) \otimes E_{1 j}\left(e \otimes x^{*}\right)$. By Proposition 4.1, $\mathfrak{A}$ satisfies Condition M2. Since $S^{k} R^{k} \rightarrow 0$ in the strong operator topology, (iii) of Proposition 1.2 is satisfied, so that $\mathfrak{A}_{\infty}$ is dense in $\mathfrak{A}$. For $a \in \mathfrak{A}_{n}$ and $\xi \in \ell_{p}(X)$ one checks that $\left\|\sum_{l=1}^{N} \sigma_{l k}(a)(\xi)\right\| \leq\|a\|\|\xi\|$ for any $k \geq n$ so that $\nu(a)=\|a\|$. Hence Condition M1 is satisfied. Finally, each change of sign and each permutation of entries in $\ell_{p}(X)$ induces an isometry, so that $\kappa(\mathcal{U})=1$, i.e., Condition M3 is fulfilled.

For a Hilbert space $H$ it was proved in W1 that $\mathcal{A}(H), \mathcal{B}(H)$, and the Calkin algebra $\mathcal{C}(H)$ are all simplicially trivial. The same argument gives

4.3 Corollary. If $X$ has the bounded approximation property, then $\mathcal{A}\left(\ell_{p}(X)\right)$, $\mathcal{B}\left(\ell_{p}(X)\right)$, and the Calkin algebra $\mathcal{C}\left(\ell_{p}(X)\right)$ are all simplicially trivial.

Proof. When $X$ has the bounded approximation property, then so does $\ell_{p}(X)$. It follows that $\mathcal{A}\left(\ell_{p}(X)\right)$ is a strongly $\mathrm{H}$-unital Banach algebra, and the sequence $\{0\} \longrightarrow \mathcal{A}\left(\ell_{p}(X)\right) \longrightarrow \mathcal{B}\left(\ell_{p}(X)\right) \longrightarrow \mathcal{C}\left(\ell_{p}(X)\right) \longrightarrow\{0\}$ is weakly split. It was noted in DGG that $\mathcal{B}\left(\ell_{p}(X)\right)$ is simplicially trivial. The rest follows from Theorem 4.2 and excision.

If $(\mu, \Omega, \Sigma)$ is a measure space such that $L_{1}(\mu, \Omega, \Sigma)$ is finite dimensional (i.e., $(\mu, \Omega, \Sigma)$ is equivalent to a finite set), then $L_{p}(\mu, X)$ is isomorphic to a finite direct sum of copies of $X$ for all $p$. Hence, if $\mathcal{A}(X)$ is strongly $\mathrm{H}$-unital, Morita equivalence gives that $\mathcal{H}^{\bullet}\left(\mathcal{A}(X), \mathcal{A}(X)^{*}\right) \cong \mathcal{H}^{\bullet}\left(\mathcal{A}\left(L_{p}(\mu, X)\right), \mathcal{A}\left(L_{p}(\mu, X)\right)^{*}\right)$.

For infinite dimensional $L_{p}$-spaces we get

4.4 Corollary. Let $(\mu, \Omega, \Sigma)$ be a measure space which is not equivalent to a finite set, and let $X$ be a Banach space with the bounded approximation property. Then $\mathcal{A}\left(L_{p}(\mu, X)\right)$ is simplicially trivial. 
Proof. In G3 it is proved that $\mathcal{A}\left(\ell_{p}(X)\right)$ and $\mathcal{A}\left(L_{p}(\mu, X)\right)$ are Morita equivalent. For Banach algebras with bounded one-sided approximate identities, bounded Hochschild homology with coefficients in essential modules is Morita invariant [G1]. The result follows then from Theorem 4.2.

\section{ACKNOWLEDGEMENT}

The author wishes to thank the referee for pointing out the usefulness of normalizing w.r.t. an amenable subalgebra. This weakened the original assumption M3 and shortened the proof of Theorem 2.1

\section{REFERENCES}

[B1] Ariel Blanco, On the weak amenability of $\mathcal{A}(X)$ and its relation with the approximation property, J. Funct. Anal. 203 (2003), 1-26. MR1996866 (2004g:47092)

[B2] Weak amenability of $\mathcal{A}(E)$ and the geometry of $E$, J. London Math. Soc. (2) 66 (2002), 721-740. MR1934302 (2003i:46044)

[CS] E. Christensen and A. M. Sinclair, On the vanishing of $H^{n}\left(\mathcal{A}, \mathcal{A}^{*}\right)$ for certain $C^{*}$ algebras, Pacific J. Math. 137 (1989), 55-63. MR0983328 (90c:46093)

[DGG] H. G. Dales, F. Ghahramani, and N. Grønbæk, Derivations into iterated duals of Banach algebras, Studia Math. 128 (1998), 19-54. MR1489459 (99g:46064)

[G1] N. Grønbæk, Morita equivalence for self-induced Banach algebras, Houston J. Math. 22 (1996), 109-140. MR1434388 (98c:46090)

[G2] Factorization and weak amenability of algebras of approximable operators, to appear in Math. Proc. R. Ir. Acad.

[G3] , Self-induced Banach algebras, Contemp. Math. 263 (2004), 129-143. MR2097956 (2005g:46093)

[G4] Amenability of weighted convolution algebras on locally compact groups, Trans. Amer. Math. Soc. 319 (1990), 765-775. MR0962282 (90j:43003)

[J] B.E. Johnson, Cohomology in Banach algebras, Mem. Amer. Math. Soc. 127 (1972). MR0374934 (51:11130)

[JKR] B.E. Johnson, R. V. Kadison and J. R. Ringrose, Cohomology of operator algebras. III. Reduction to normal cohomology, Bull. Soc. Math. France 100 (1972), 73-96. MR 0318908 (47:7454)

[L] J.-L. Loday, Cyclic Homology, Grundlehren der mathematischen Wissenschaften, Springer-Verlag, Berlin, Heidelberg, 1992. MR1217970 (94a:19004)

[W1] M. Wodzicki, The long exact sequence in cyclic homology associated with an extension of algebras, C. R. Acad. Sci. Paris Sér. A-B 306 (1988), 399-403. MR0934604 (89i:18012)

[W2] Vanishing of cyclic homology of stable $C^{*}$-algebras, C. R. Acad. Sci. Paris Sér. I 307 (1988), 329-334. MR0958792 (89j:46069)

[W3] Homological properties of rings of functional-analytic type, Proc. Natl. Acad. Sci. USA 87 (1990), 4910-4911. MR.1058786 (91j:19011)

Department of Mathematics, University of Copenhagen, Universitetsparken 5, DK2100 Copenhagen $\varnothing$, Denmark

E-mail address: gronbaek@math.ku.dk 